\author{
Eva Espinar-Ruiz \\ Universidad de Alicante - España
}

Begoña López-Monsalve

Universidad de Alicante - España

\title{
Infancia y violencia de género: cuantificación y percepciones
}

\begin{abstract}
Resumen: Cada vez más autores destacan la necesaria incorporación de la infancia en el estudio de la violencia de género y, en concreto, de la violencia contra la mujer por parte del compañero íntimo (VCl). Tal incorporación puede facilitar no sólo una menor invisibilidad y desprotección de niños y niñas, sino también una mejor comprensión de la propia violencia, sus características y dinámicas. Partiendo de estas consideraciones, en este artículo se presentan los resultados de un análisis secundario de los datos obtenidos en la última macroencuesta sobre violencia contra las mujeres en España. La información disponible permite analizar: 1) la presencia de menores expuestos a VCl; 2) la posible relación entre esta presencia y la formulación de denuncias; $y$ 3) la percepción que tienen las mujeres víctimas de VCl en torno al rol parental de los agresores.
\end{abstract}

Palabras clave: violencia de género, violencia del compañero íntimo, infancia, análisis cuantitativo, rol parental.

Copyright @ 2015 by Revista Estudos Feministas.

' Kathya ARAUJO, Virginia GUZMÁN y Amalia MAURO, 2000.

${ }^{2}$ Claire M. RENZETTI, Jeffrey L. EDLESON y Raquel K. BERGEN, 2011; Mary R. JACKMAN, 2006.

\section{Introducción}

La falta de reconocimiento social refuerza los procesos de victimización en los casos de violencia y, en concreto, cuando nos enfrentamos a la violencia de género, un problema presente a lo largo de la historia pero que no ha sido considerado como tal hasta hace relativamente poco tiempo'. Así, ha sido en las últimas décadas, y gracias a la promoción desde las instituciones, los movimientos sociales y el pensamiento feminista, cuando se ha desarrollado una importante labor de investigación, difusión y denuncia en torno a la violencia de género. Como resultado, podemos hablar, actualmente, de la existencia de numerosas investigaciones cuantitativas y cualitativas, a nivel nacional e internacional ${ }^{2}$, que han venido acompañadas, en mayor 0 
${ }^{3}$ R. Amy ELMAN, 2013.

${ }^{4}$ Los Informes del Observatorio (dependiente del Gobierno de España) están disponibles en: ww w. ms s si.gob.es/s s i/ violenciaGenero. Acceso en: 02/ 04/2014.

${ }^{5}$ Elena AYLLÓN, 2011.

${ }^{6}$ Beatriz ATENCIANO, 2009.

${ }^{7}$ Maria ERIKSSON, 2010.

${ }^{8}$ Macroencuesta encargada por el desaparecido Ministerio de Igualdad. Constituye el estudio $\mathrm{n}^{\circ}$ 2.858 del CIS. Puede consultarse información adicional en: www.cis.es. Acceso en: 28/03/ 2014.

${ }^{9}$ Aunque la información referida a menores expuestos a $\mathrm{VCl}$ en la encuesta es limitada, consideramos justificado su análisis dado que se trata del único estudio representativo a nivel nacional y con una muestra suficientemente elevada como para poder trabajar estadísticamente con los porcentajes de mujeres víctimas de $\mathrm{VCl}$.

452 menor medida, de cambios legislativos y en la política social de los distintos estados ${ }^{3}$.

Entre las diferentes formas en que puede materializarse la violencia de género, ha recibido especial atención el estudio de la violencia contra las mujeres por parte del compañero íntimo ( $\mathrm{VCl}$ ), cuyo análisis suele situarse en el marco de relaciones heterosexuales y en la violencia ejercida por la pareja o ex-pareja sentimental de la mujer. Esta violencia es también el objeto de estudio del presente artículo, si bien para centrarnos en el análisis de una serie de aspectos relacionados con el colectivo de niños y niñas expuestos a la misma. En este sentido, y a pesar de que organismos como el Observatorio Estatal de Violencia sobre la Mujer ${ }^{4}$ en España o la organización Save the Children ${ }^{5}$ destacan los efectos que la $\mathrm{VCl}$ tiene sobre la infancia, son relativamente pocas las investigaciones que centran su atención en esta población'. Especialmente escasos son los estudios que intentan combinar la redefinición de los menores expuestos a VCl como víctimas de esta violencia con un análisis de género, que pueda facilitar una mayor comprensión tanto de la situación de niñas y niños, como de las propias dinámicas de la violencia, de las relaciones entre padres/madres e hijos/as en el contexto de VIC y de la construcción social de tales relaciones ${ }^{7}$.

Partiendo de estas consideraciones, en los siguientes apartados presentamos los resultados de un análisis secundario de los datos obtenidos por el Centro de Investigación Sociológicas (CIS) en la última macroencuesta sobre violencia contra las mujeres en España, realizada en el $2011^{8}$. En concreto, y teniendo en cuenta que el propósito central de esta encuesta no es el análisis de los niños y niñas expuestos a $\mathrm{VCl}$, la explotación de los datos se orienta a extraer y analizar toda aquella información que puede relacionarse con esta población?. Así, nuestros objetivos son: determinar la presencia de menores en contextos de $\mathrm{VCl}$; analizar la posible relación entre esta presencia y un elemento clave en la propia dinámica de la violencia como es la decisión por parte de la mujer de denunciar; y estudiar la percepción que tienen las mujeres víctimas de $\mathrm{VCl}$ sobre el rol parental de los agresores. Las hipótesis de las que partimos plantean que la presencia de hijos en una relación donde se ejerce $\mathrm{VCl}$ condiciona a la mujer de manera positiva a la hora de efectuar la denuncia y que existe una tendencia a disociar la violencia hacia la pareja del rol parental, de manera que los comportamientos violentos hacia la madre no determinan la percepción que se tiene de las habilidades parentales del agresor. 
${ }^{10}$ Eva ESPINAR y Miguel Ángel MATEO, 2007, p. 193.

"Resolución del Parlamento Europeo sobre tolerancia cero ante la violencia contra las mujeres de 16 de octubre de 1997 (Diario Oficial $n^{\circ} \mathrm{C} 304$, p. 0055). Localizable en: http://eur-lex.europa.eu. Acceso en: 02/04/2014.

${ }^{12}$ Esperanza MARTíN y Manuel MARTíN, 1999, p. 19.

${ }^{13}$ WHO, 2013

${ }^{14}$ FRA, 2014.

${ }^{15}$ Isabel RUIZ, Juncal PLAZAOLA Carmen VIVES, M. Isabel MONTERO, Vicenta ESCRIBÀ, Esther JIMÉNEZ Y David MARTíN, 2010.

16 Carolina ØVERLIEN, 2010; ERIKSON, 2010.

17 Karin GRIP, 2012; Stephanie HOLT, Helen BUCKLEY y Sadhbh WHELAN, 2008.

\section{Marco teórico}

El término violencia de género hace referencia a aquellas formas de violencia que hunden sus raíces en las definiciones y relaciones de género dominantes en una sociedad dada ${ }^{10}$. Es decir, se trata de una violencia vinculada, tanto a los desequilibrios "en las relaciones de poder entre los sexos en los ámbitos social, económico, religioso o político"11. como a unas concretas definiciones, estereotipos, identidades y expectativas sobre lo que supone ser hombre y lo que supone ser mujer. Entre las diferentes formas que puede adoptar la violencia de género en este artículo, nos centraremos en una de ellas: la violencia contra las mujeres en el seno de una relación de pareja, haya o no convivencia (esposos, compañeros, novios, ya lo sean actualmente o lo hayan sido en el pasado), que denominaremos violencia del compañero íntimo (VCl). En este sentido, suele reconocerse que "las agresiones que sufren las mujeres de sus esposos o compañeros ofrecen la máxima evidencia posible del papel tan determinante que tienen las desigualdades basadas en el género"12.

Esta violencia viene recibiendo una creciente atención y en torno a ella existe un relativamente amplio acuerdo terminológico y en cuanto a efectos, características y grado de extensión. En concreto, según la Organización Mundial de la Salud alrededor de un $23 \%$ de las mujeres que viven en países de altos ingresos y que alguna vez han tenido pareja han sufrido en algún momento de su vida violencia física y/o sexual por parte de un compañero íntimo ${ }^{13}$. En el conjunto de la Unión Europea, en torno al $22 \%$ de las mujeres adultas declaran haber sufrido $\mathrm{VCl}$ (física y/o sexual) alguna vez en su vida ${ }^{14}$. En España, un estudio realizado con una muestra de 11.000 mujeres usuarias de los centros de atención primaria registró una prevalencia del $35 \%$ para el año $2007^{15}$

Muchas de las investigaciones sobre $\mathrm{VCl}$ incorporan en su análisis, en mayor o menor medida, referencias a los niños y niñas implicados, aunque, normalmente, éstos son objeto de estudio sólo de forma indirecta. Por ello, y a pesar de un creciente interés en los últimos años, algunos autores siguen calificando de insuficiente la información disponible sobre menores expuestos a $\mathrm{VCl}^{16}$. Muestra de estas limitaciones es la amplia diversidad de términos empleados para referirse a este colectivo. En concreto, se han ido abandonando algunas expresiones como es el caso de "testigos de VCl", que difícilmente incluye la posibilidad de oír, ser parte o ver las consecuencias de la violencia, como heridas, moratones o mobiliario destrozado ${ }^{17}$. La opción se abre a otros términos más inclusivos que pretenden hacer 


\author{
18 George W. HOLDEN, Robert A. \\ GEFFNER y Ernest N. JOURILES, \\ 1998. \\ ${ }^{19}$ Chris GODDARD y Gillinder BEDI, \\ 2010 \\ ${ }^{20}$ ERIKSSON, 2010. \\ ${ }^{21}$ Alison CUNNINGHAM y Linda \\ BAKER, 2004; Maria ERIKSSON, \\ 2011.
}

${ }^{22} \varnothing$ VERLIEN, 2010, p. 87.

referencia a todos los niños y niñas que viven en un hogar donde existe $\mathrm{VCl}$ y que intentan abarcar sus diversas experiencias: "expuestos a"18, "forzados a vivir con"19 o "sometidos a"20 violencia. Siguiendo esta lógica, algunos autores plantean la necesidad de incluir en la categoría de víctimas al conjunto de menores expuestos a $\mathrm{VCl}$, independientemente de que sean o no objeto directo de violencia, con las implicaciones prácticas y políticas que tal calificación supone ${ }^{21}$.

Entre las investigaciones llevadas a cabo predomina el uso de metodologías cuantitativas, fundamentalmente orientadas a la descripción de los efectos que la exposición a la violencia tiene para los menores ${ }^{22}$. De esta forma, es relativamente amplia la información existente en torno a los efectos físicos, psicológicos, emocionales y de comportamiento que tiene para niños y niñas vivir en contextos de $\mathrm{VCl}^{23}$. Entre estos efectos, destaca la mayor probabilidad de verse envueltos en situaciones de violencia presente y futura (como víctimas o agresores), incluida $\mathrm{VCl}^{24}$; así como de sufrir abusos físicos o sexuale ${ }^{25}$ y abandono o desatención por parte de sus progenitores ${ }^{26}$. Los resultados obtenidos hacen concluir a $\varnothing$ verline ${ }^{27}$ que el impacto que tiene sobre los menores la exposición a $\mathrm{VCl}$ es mayor de lo que tradicionalmente se ha considerado.

Además de la identificación de los efectos de la $\mathrm{VCl}$ sobre niñas y niños, algunos estudios han tratado, también, de cuantificar su grado de extensión. Sin embargo, tal y como destaca $\varnothing$ verline ${ }^{28}$, "both because of the variability in what is understood and reported as domestic violence, and because researchers and practitioners define 'a child exposed to domestic violence' differently" la comparación entre los resultados disponibles es extremadamente difícil. A pesar de ello, conviene mencionar algunos datos. Así, por ejemplo, en la encuesta de Annebarck et al. ${ }^{29}$, a partir de una muestra de jóvenes suecos de entre 13 y 17 años, un $11 \%$ respondió afirmativamente al ser cuestionado sobre la exposición a cualquier incidente de $\mathrm{VCl}$. Para los Estados Unidos, Zinzow et al.$^{30}$ obtuvieron un porcentaje de exposición a formas severas de $\mathrm{VCl}$ del $9 \%$ entre adolescentes de 12 a 17 años; mientras que una investigación realizada en el Reino Unido ${ }^{31}$ concluye que un $12 \%$ de los niños menores de 11 años y un $17 \%$ de los adolescentes de entre 11 y 17 años se han visto expuestos al menos en una ocasión a VCl física.

Otras dimensiones y perspectivas también han sido analizadas, aunque en menor medida. Este es el caso del significado que la $\mathrm{VCl}$ tiene para los propios menores, la inclusión de la perspectiva de género o el estudio de la interpretación que la madre hace de la exposición a VCl de 
32 ERIKSSON, 2010; GuY PEREL Y Einat PELED, 2008; Emily F. ROTHMAN, David G. MANDEL y Jay G. SILVERMAN, 2007

${ }^{33}$ Maria ERIKSSON, 2002, p. 3.

${ }^{34}$ Maria ERIKSSON y Marianne HESTER, 2001; ERIKSSON, 2002. sus hijos e hijas. Entre los temas más desatendidos destaca el análisis de la relación entre los menores y el padre agresor, así como la percepción que éste último tiene de la violencia que ejerce y de su rol parental ${ }^{32}$. En palabras de Eriksson ${ }^{33}$ : "A man's relationship with his partner and his relationships with his children can be conceptualized as parts of the same gender project, as arenas that might be linked to an overall process of gender constitution". Sin embargo, los estudios realizados en este campo hablan de una tendencia generalizada a desligar la figura del agresor de su rol parental, de forma que los padres son, en términos generales, interpretados como esencialmente no violentos ${ }^{34}$.

En este artículo intentaremos realizar algunas aportaciones en este área de estudio, a pesar de las limitaciones que impone el análisis basado en datos secundarios, que, por ejemplo, no nos permitirá atender a las experiencias de los menores, ni a las percepciones por parte de los agresores. Sin embargo, sí podremos aportar información sobre otras cuestiones relevantes: la presencia de hijos entre los casos de $\mathrm{VCl}$; la percepción que la madre tiene del rol parental del agresor; en qué medida las mujeres víctimas de $\mathrm{VCl}$ afirman que sus hijos se ven afectados por la misma; y hasta qué punto la presencia de hijos puede incrementar las probabilidades de que la madre denuncie al agresor.

\section{Metodología}

Para la elaboración de este artículo se ha desarrollado un análisis secundario del estudio $n^{\circ} 2.858$ del CIS, realizado en el año 2011 por encargo de la Delegación del Gobierno para la Violencia de Género. En concreto, este estudio constituye la cuarta macroencuesta sobre violencia contra las mujeres en España y su principal diferencia respecto a las tres anteriores (realizadas en 1999, 2002 y 2006) reside en el modo de aplicación. Mientras que en las primeras se entrevistaba telefónicamente, el último estudio se basó en entrevistas presenciales en el domicilio de las encuestadas. Este cambio ha implicado una disminución del tamaño de la muestra (de 20.552 en la primera macroencuesta a 7.898 en la última), en todo caso representativa del conjunto de mujeres residentes en España mayores de 18 años.

El principal objetivo de la serie de macroencuestas es conocer el número aproximado de mujeres que son o han sido víctimas de violencia en el ámbito familiar, con especial atención a la VCl. Adicionalmente, se incluyen una serie de variables que permiten analizar la posible relación entre diferentes características y tal victimización. En este sentido, en la encuesta de 2011 se introdujeron algunas modificaciones en el cuestionario que facilitan la obtención de 
${ }^{35}$ Tal y como recoge el informe elaborado por Sigma Dos (2006) en relación a la llI Macroencuesta sobre la violencia contra las mujeres. Informe localizable en: http://www.unece.org. Acceso en: 02/04/2014. información sobre la existencia o no de hijos e hijas expuestos a VCl, así como en torno a la percepción del rol parental y de los derechos de custodia del padre agresor por parte de las mujeres. Son estas variables las que centrarán nuestra atención.

En concreto, los datos ofrecidos por el CIS han sido tratados haciendo uso del programa estadístico SPSS, que ha permitido el análisis univariable y bivariable de la información relevante. Dada la naturaleza fundamentalmente nominal de las variables seleccionadas, se ha optado por la aplicación de un análisis exploratorio y descriptivo, explorando las posibles relaciones a través de tablas de contingencia, y haciendo uso de los resultados de Chicuadrado y de los residuos tipificados corregidos, que permiten analizar no sólo la relación entre variables, sino también entre categorías concretas de las variables.

Ahora bien, el primer paso necesario antes de elaborar los posteriores análisis ha sido la construcción de indicadores de $\mathrm{VCl}$. En este sentido, en la serie de macroencuestas sobre violencia contra las mujeres se han venido aplicando dos estrategias diferentes para detectar casos de violencia, resumidas con los términos: violencia tipo A y violencia tipo B. En la categoría de violencia tipo A se sitúan las mujeres "que han sido consideradas técnicamente como maltratadas"; mientras que bajo el epígrafe de violencia tipo B se incluyen a las "mujeres que se autoclasifican como maltratadas" 35 .

De esta forma, la violencia tipo A hace referencia a aquellas mujeres calificadas como víctimas por su respuesta afirmativa ante una serie de ítems que reflejan diferentes formas de violencia. Esta clasificación se realiza independientemente de la interpretación que las propias mujeres puedan hacer de su experiencia. Es decir, se considera que sufren violencia aunque ellas puedan no reconocerse como víctimas. En el cuestionario aplicado en el 2011 , la pregunta concreta que se formuló fue: (P35) "¿En la actualidad, con qué frecuencia diría usted que una persona de su entorno cercano...?". La respuesta estaba asociada a una batería de 26 ítems, que incluyen diferentes manifestaciones de violencia psicológica, física, sexual, económica o social. La información disponible permite identificar a la persona que ejerce tal violencia y, por tanto, seleccionar aquellos casos en los que ésta es el marido/pareja o el exmarido/expareja.

En los informes correspondientes a las tres primeras macroencuestas, se seleccionaron las respuestas a 13 de los 26 ítems (aquellos que describen situaciones que son calificadas como de mayor gravedad) para la construcción del indicador de violencia tipo $A$, considerando las categorías "frecuentemente" o "a veces". Esta misma estrate- 
${ }^{36}$ Cabe destacar que en los diferentes informes de resultados de la macroencuesta del 2011 (tanto el elaborado por el $\mathrm{Cls}$ como el de la Delegación de Gobierno para la Violencia de Género) no se ha construido el indicador de violencia tipo $A, y$ simplemente se reflejan las respuestas para cada ítem de forma desglosada.

${ }^{37}$ SIGMA DOS, 2006.

\footnotetext{
${ }^{38} \varnothing$ VERLINE, 2010.
}

gia es la que se ha seguido en la presente investigación a la hora de analizar los resultados de la cuarta macroencuesta ${ }^{36}$. De esta forma, y teniendo en cuenta únicamente aquellos casos en los que la violencia es ejercida por parejas o exparejas de la mujer ( $\mathrm{VCl}$ ), podemos hablar de un 10,2\% del total de mujeres encuestadas víctimas de violencia tipo $\mathrm{A}$. Así, los resultados obtenidos se aproximan a los que arrojan las macroencuestas previas (con un $12,4 \%$ en 1999 , un $11,1 \%$ en el 2002 y un $9,6 \%$ en el 2006$)^{37}$.

Respecto a la segunda estrategia, la violencia tipo B, consiste en preguntar directamente a la mujer consultada si durante el último año (P36) o alguna vez en su vida (P37) han "sufrido alguna situación por la que se haya considerado maltratada" por parte de su marido/pareja, exmarido/expareja u otro/a familiar. Así, la violencia tipo B hace referencia a aquellas situaciones en las que la propia mujer percibe que ha sido víctima de maltrato; es decir, se autoclasifica como tal. Una vez más es posible identificar a aquellas mujeres que respondieron afirmativamente y seleccionaron como agresores a sus parejas o exparejas. En este caso, son un 2,9\% las mujeres que afirman ser víctimas de $\mathrm{VCl}$ en el último año y un 10,7\% las que afirma haberlo sido alguna vez en su vida.

\section{Resultados}

\subsection{Menores expuestos a VCl}

La falta de acuerdo terminológico respecto a qué menores contar y las dificultades a la hora de detectar y contabilizar los casos de VCI hacen especialmente difícil la obtención de datos comparables en torno al número de niñas y niños expuestos a esta forma de violencia ${ }^{38}$. Usualmente, es la perspectiva de la madre la que se toma como referencia para analizar la presencia de $\mathrm{VCl}$ y el grado en que los menores se ven implicados. Con ello, los intentos de contabilizar dependen, en gran medida, de la percepción que la propia mujer tiene tanto de la violencia como de sus efectos.

Los datos que ofrece la macroencuesta del CIS permiten seguir dos procedimientos en el intento de determinar cuantitativamente la presencia de menores en contextos de $\mathrm{VCl}$, haciendo uso de los dos indicadores de violencia construidos: violencia tipo $\mathrm{A}$ (mujeres consideradas técnicamente como maltratadas) y violencia tipo B (mujeres que se autoclasifican como maltratadas). Así, podemos destacar que en un $43 \%$ de los casos de violencia tipo A hay hijos menores; mientras que un $59 \%$ de las mujeres que se han sentido maltratadas en el último año y un $54 \%$ de las que se han sentido maltratadas alguna vez en su vida afirman haber estado conviviendo con hijos menores cuando 
${ }^{39}$ Los datos disponibles hacen únicamente referencia a la presencia de hijos, pudiendo quedar fuera del recuento menores que mantengan otro tipo de relación con la mujer encuestada.

${ }^{40}$ ERIKSSON y HESTER, 2001.

${ }^{41} \varnothing$ VERLINE, 2010, p. 89.

${ }^{42}$ Eva ESPINAR, 2006.

${ }^{43}$ Kerstin EDIN, Lars DAHLGREN, Ann LALOS y UIf HÖGBERG, 2010; Mariano SALAZAR, Ulf HÖGBERG, Eliette VALLADARES y Ann ÖHMAN 2012.

${ }^{44}$ Judy C. CHANG, Diane DADO Lynn HAWKER, Patricia CLUSS Raquel BURANOSKY, Leslie SLAGEL, Melissa MCNEIL y Sarah $\mathrm{H}$. SCHOLLE, 2010; Viveka ENANDER y Carin HOLMBERG, 2008. tal situación se produjo ${ }^{39}$. Estos resultados respaldan el posicionamiento de autores previos ${ }^{40}$ que señalan la relevancia de incluir a la infancia en el estudio de la $\mathrm{VCl}$.

A las mujeres que se autoclasificaron como maltratadas (violencia tipo B) y que tenían hijos menores en el momento del maltrato se les preguntó si sus hijos habían padecido, directamente, situaciones de violencia. Ante tal posibilidad, respondió afirmativamente un $54 \%$ de aquellas que se habían sentido maltratadas alguna vez y un $60 \%$ de las que se habían sentido maltratadas durante el último año. Estos resultados no permiten saber qué entienden las mujeres encuestadas por "padecer directamente situaciones de violencia" y hasta qué punto consideran que la mera presencia en contextos de VCl puede afectar a sus hijos. De esta forma, aunque los porcentajes obtenidos son relativamente elevados, no dejan de respaldar las conclusiones de estudios anteriores que destacan una tendencia a la subpercepción por parte de las madres del grado en que sus hijos se pueden ver afectados por la violencia que ellas mismas padecen ${ }^{41}$.

\subsection{Presencia de hijos y formulación de denuncias}

Investigaciones previas destacan el efecto contradictorio que la presencia de hijos tiene en la interpretación de la VCl por parte de la mujer, así como en su decisión a la hora de poner fin o proseguir una relación marcada por la violencia $^{42}$. Esta presencia puede, tanto fomentar la permanencia en la relación de pareja, anteponiendo (al menos desde el punto de vista de las protagonistas) el bienestar de los hijos al suyo propio ${ }^{43}$, como constituirse en incentivo a la hora de formular denuncias o poner fin a esa relación, especialmente cuando la mujer percibe que los hijos se convierten o corren el peligro de convertirse en víctimas directas de la violencia física del agresor ${ }^{44}$.

Con los datos de la macroencuesta del CIS, no es posible profundizar en el estudio de todos estos aspectos, pero sí podemos analizar la relación entre dos variables fundamentales: la presencia de hijos menores en los contextos de $\mathrm{VCl}$ y la formulación de denuncias. De esta forma, para los casos de violencia tipo B, es decir para aquellas mujeres que se autoclasifican como maltratadas, se ha podido analizar si es mayor la probabilidad de denunciar cuando en el momento del maltrato existen hijos menores (ver tabla 1). 
TABLA 1. Tabla de contingencia entre formulación de denuncias y presencia de hijos menores de edad (\%)

Base: Mujeres que afirman haberse sentido maltratadas alguna vez (violencia tipo B)

\begin{tabular}{|l|l|l|l|l|}
\hline \multicolumn{2}{|c|}{} & \multicolumn{2}{|l|}{ Presencia de hijos menores } & \multirow{2}{*}{ Total } \\
\cline { 3 - 5 } Formulación de denuncia & Sí & 36,0 & 21,3 & 30,9 \\
\cline { 3 - 5 } & No & 64,0 & 78,7 & 69,1 \\
\hline Total & 100,0 & 100,0 & 100 \\
\hline
\end{tabular}

Fuente: Elaboración propia a partir de los resultados del estudio $n^{\circ} 2.848$ del CIS

* Valor Chi- cuadrado de Pearson 16,145; significación asintótica bilateral 0,000

Tal y como muestra el Chi-cuadrado de Pearson asociado a la tabla 1 , es posible hablar de una relación estadísticamente significativa entre ambas variables. Es decir, la probabilidad de denunciar es significativamente mayor cuando hay hijos menores de edad. Así, un 36\% de las mujeres con hijos menores en el momento del maltrato denunciaron al agresor, frente al $21 \%$ de las que no tenían hijos o estos no eran menores de edad. En definitiva, la presencia de hijos parece constituir un estímulo a la hora de denunciar. Aun así, es necesario destacar que la mayor parte de las mujeres no denuncian al agresor (un 69\%), ya tengan hijos menores en el momento del maltrato (un 64\%) o no (un 79\%).

La siguiente posibilidad que permite analizar la macroencuesta, siempre para la violencia tipo B, es si el hecho de que los hijos se conviertan en víctimas directas de las agresiones (o al menos que la encuestada lo perciba de esta forma) incentiva a la mujer a denunciar la situación. Los resultados pueden observarse en la tabla 2 . También en esta ocasión el Chi-cuadrado muestra una relación significativa entre la presentación de denuncias y la percepción de los hijos como afectados directamente por la violencia. Un $47 \%$ de las mujeres que afirmaron que sus hijos habían padecido situaciones de violencia denunciaron; frente al $24 \%$ de las que lo negaron. Aun así, podemos seguir destacando en torno a un $54 \%$ de casos con hijos menores víctimas directas de violencia en los que no hubo denuncia.

A aquellas mujeres víctimas de violencia tipo B y que no habían denunciado al agresor se les preguntó por las razones que las llevaron a no denunciar. Un $40 \%$ de estas mujeres señaló, como motivo (ya fuera en primer, segundo o tercer lugar), "por mis hijos". Los datos disponibles no permiten conocer si esta opción responde a la búsqueda de una seguridad económica para los hijos, o tiene más que ver con mantener la unidad familiar y la convivencia del progenitor con éstos. En todo caso, los datos suponen un respaldo a resultados previos, aparentemente contradictorios, 
en los que los hijos son a un mismo tiempo incentivo para denunciar y poner fin a una relación de pareja y factor para no denunciar e intentar mantener la unidad familiar. Igualmente, parecen indicar una limitada percepción por parte de las mujeres de los riesgos que para los niños y niñas puede suponer la exposición a contextos de $\mathrm{VCl}$.

TABLA 2. Tabla de contingencia entre formulación de denuncias y presencia de hijos menores víctimas de agresiones (\%)

Base: Mujeres que afirman haberse sentido maltratadas alguna vez (violencia tipo B) y tenían hijos menores en el momento del maltrato

\begin{tabular}{|l|l|l|l|l}
\hline \multicolumn{2}{|c|}{} & \multicolumn{2}{|l|}{$\begin{array}{l}\text { Presencia de hijos menores que han } \\
\text { padecido directamente situaciones } \\
\text { de violencia }\end{array}$} & \multirow{2}{*}{ Total } \\
\cline { 3 - 4 } \multicolumn{2}{l|}{} & Sí & No & \\
\hline \multirow{2}{*}{ Formulación de denuncia } & Sí & 46,5 & 24,4 & 36,5 \\
\cline { 2 - 5 } & No & 53,5 & 75,6 & 63,5 \\
\hline \multicolumn{2}{l|l}{ Total } & 100,0 & 100,0 & 100,0 \\
\hline
\end{tabular}

Fuente: Elaboración propia a partir de los resultados del estudio n 2.848 del CIS

* Valor de Chi cuadrado de Pearson 23, 235; significación asintótica bilateral 0,000

${ }^{45}$ ERIKSSON, 2002; Lara GUILLE, 2004; PEREL Y PELED, 2008 ; ROTHMAN, MANDEL Y SILVERMAN, 2007; Emily J. SALISBURY, Kris HENNING y Robert HOLDFORD, 2009.

${ }^{46}$ En las tablas 3, 4 y 5 se incluye entre paréntesis los residuos tipificados corregidos. Un valor superior $a \pm 1,9$ indica una relación significativa (positiva o negativa según el signo del valor) entre el par de categorías correspondientes. De esta forma, se facilita la interpretación de las tablas y el análisis de la relación entre las variables, más allá de lo que pueda indicar el estadístico Chi-cuadrado.

\section{3. La custodia y la figura paterna en los casos de $\mathrm{VCl}$}

Según diversos autores ${ }^{45}$, existe una tendencia generalizada a disociar el rol paterno del rol de pareja, de manera que la presencia de actitudes o comportamientos violentos hacia la madre no implica, necesariamente, una desvalorización de las habilidades parentales del agresor. Esta disociación estaría presente tanto entre las víctimas de $\mathrm{VCl}$, como en la sociedad en general o en el ámbito asistencial y judicial, con una tendencia a anteponer el mantenimiento de la relación entre el padre agresor y sus hijos frente a otras consideraciones.

A través de la encuesta que venimos analizando es posible estudiar, directamente, este punto, ya que se incluye una pregunta sobre el grado de acuerdo con la siguiente afirmación: "Que un hombre maltrate a su pareja no tiene por qué implicar que sea un mal padre". Los resultados que se obtienen para el conjunto de la muestra parecen contradecir la supuestamente generalizada tendencia a disociar los roles de pareja y de progenitor. Así, el $69 \%$ de las encuestadas está poco o nada de acuerdo con la afirmación, mientras que sólo el $23 \%$ está mucho o bastante de acuerdo. Ahora bien, si se observan los resultados de la tabla $3^{46}$, llama la atención que, de forma estadísticamente 
significativa, son las mujeres víctimas de $\mathrm{VCl}$, tanto tipo $\mathrm{A}$ como tipo B, las que en mayor medida están muy o bastante de acuerdo con la disociación de los roles de pareja (agresor) y de padre.

TABLA 3. Tabla de contingencia entre violencia (tipo A y tipo B) y disociación de roles (pareja/

\begin{tabular}{|c|c|c|c|c|c|}
\hline & \multicolumn{2}{|c|}{ Violencia tipo $A^{*}$} & \multicolumn{2}{|c|}{ Violencia tipo B** } \\
\hline & & No & Sí & No & Sí \\
\hline \multirow{3}{*}{$\begin{array}{l}\text { Grado de acuerdo con la } \\
\text { afirmación: "Que un hombre } \\
\text { maltrate a su pareja no tiene } \\
\text { por qué implicar que sea un } \\
\text { mal padre" }\end{array}$} & Mucho/Bastante & $\begin{array}{l}22,0 \\
(-4,6)\end{array}$ & $\begin{array}{l}29,1 \\
(4,6)\end{array}$ & $\begin{array}{l}22,4 \\
(-2,1)\end{array}$ & $\begin{array}{l}25,6 \\
(2,1)\end{array}$ \\
\hline & Poco/Nada & $\begin{array}{l}69,6 \\
(4,1)\end{array}$ & $\begin{array}{l}62,5 \\
(-4,1)\end{array}$ & $\begin{array}{l}68,6 \\
(-1,3) \\
\end{array}$ & $\begin{array}{l}70,8 \\
(1,3)\end{array}$ \\
\hline & NS/NC & $\begin{array}{l}8,4 \\
(0,0)\end{array}$ & $\begin{array}{l}8,4 \\
(0,0)\end{array}$ & $\begin{array}{l}9,0 \\
(5,3)\end{array}$ & $\begin{array}{l}3,7 \\
(-5,3)\end{array}$ \\
\hline \multicolumn{2}{|l|}{ Total } & 100,0 & 100,0 & 100,0 & 100,0 \\
\hline
\end{tabular}

Fuente: Elaboración propia a partir de los resultados del estudio $\mathrm{n}^{\circ} 2.848 \mathrm{del} \mathrm{CIS}$

*Valor de Chi cuatro de Pearson 21,353; significación asintótica bilateral 0,000.

${ }^{47}$ Valor de Chi-cuadrado de Pearson 4,800. Significación 0,028 .
Teniendo en cuenta únicamente a aquellas mujeres que se han sentido maltratadas alguna vez en su vida (violencia tipo B), no se observan diferencias significativas a la hora de disociar roles entre las que tenían hijos menores en el momento del maltrato y las que no. Sin embargo, las diferencias sí son significativas entre las que afirmaron que sus hijos habían padecido directamente situaciones de violencia y aquellas que lo negaron ${ }^{47}$. De esta forma, respecto a si un hombre que maltrata puede ser un buen padre, el $76 \%$ de las mujeres maltratadas que tenían hijos que, según ellas, también habían sido víctimas directas de la violencia, se mostraron poco o nada de acuerdo con tal posibilidad. Este porcentaje es significativamente menor en el caso de las mujeres que afirmaron que sus hijos no habían sufrido la violencia directa (desciende hasta el $66 \%$ ). Las preguntas incluidas en el cuestionario no nos permiten indagar en mayor profundidad sobre qué entiende la madre por el hecho de que sus hijos hayan padecido o no directamente situaciones de violencia, pero no deja de ser destacable que un $24 \%$ de las mujeres que afirman que sus hijos las padecieron están muy o bastante de acuerdo con la afirmación que supone disociar los roles de pareja-agresor y padre. Es un porcentaje significativamente menor al de los casos con hijos no directamente afectados (siempre según la percepción de la madre), pero que indica una cierta aceptación de la separación de roles entre mujeres que afirman que el agresor también lo era de sus hijos.

Otras dos variables nos pueden, indirectamente, ofrecer información sobre la percepción del rol parental del 
agresor por parte de las mujeres. Así, en el estudio del CIS también se pregunta por el grado de acuerdo con las siguientes afirmaciones, relativas a la custodia de los menores:

- Si hay sentencia condenatoria firme, se debe quitar la custodia de los menores al maltratador.

- Después de cumplida la condena, debería ser devuelta la custodia al padre.

En el caso del primer ítem, los resultados difieren, hasta cierto punto, con respecto a los vistos para la afirmación anterior. En primer lugar, el rechazo a la disociación de roles se eleva hasta el $83 \%$ en el conjunto de la muestra (siendo ese el porcentaje de mujeres que están muy o bastante de acuerdo con la retirada de custodia de los hijos al maltratador en el caso de sentencia condenatoria firme). Igualmente, la tabla 4 muestra, por un lado, una relación significativa entre ser víctima de violencia tipo A y un mayor desacuerdo con la retirada de la custodia al agresor (en coherencia con los datos de la tabla 3); así como una relación también significativa entre haberse sentido maltratada (violencia tipo B) y un mayor acuerdo con la retirada de la custodia. En este sentido, la presencia de una resolución legal (una sentencia condenatoria) parece incrementar la asociación de roles (pareja-agresor y padre), especialmente en el caso de aquellas mujeres que afirman haberse sentido maltratadas.

TABLA 4. Tabla de contingencia entre violencia (tipo A y tipo B) y opinión sobre la retirada de la custodia al maltratador (\%)

\begin{tabular}{|c|c|c|c|c|c|}
\hline & & \multicolumn{2}{|c|}{ Violencia tipo $A^{*}$} & \multicolumn{2}{|c|}{ Violencia tipo $B^{* *}$} \\
\hline & & No & Sí & No & Sí \\
\hline \multirow{3}{*}{$\begin{array}{l}\text { Grado de acuerdo con la } \\
\text { afirmación: "Si hay sentencia } \\
\text { condenatoria firme se debe quitar la } \\
\text { custodia de los menores al } \\
\text { maltratador" }\end{array}$} & Mucho/Bastante & $\begin{array}{l}83,4 \\
(1,5)\end{array}$ & $\begin{array}{l}81,3 \\
(-1,5)\end{array}$ & $\begin{array}{l}82,8 \\
(-2,9)\end{array}$ & $\begin{array}{l}86,7 \\
(2,9)\end{array}$ \\
\hline & Poco/Nada & $\begin{array}{l}7,9 \\
(-3,0)\end{array}$ & $\begin{array}{l}11,0 \\
(3,0)\end{array}$ & $\begin{array}{l}8,2 \\
(-0,4)\end{array}$ & $\begin{array}{l}8,6 \\
(0,4)\end{array}$ \\
\hline & NS/NC & $\begin{array}{l}8,7 \\
(1,0)\end{array}$ & $\begin{array}{l}7,7 \\
(-1,0)\end{array}$ & $\begin{array}{l}9,1 \\
(4,3)\end{array}$ & $\begin{array}{l}4,7 \\
(-4,3)\end{array}$ \\
\hline \multicolumn{2}{|l|}{ Total } & 100,0 & 100,0 & 100,0 & 100,0 \\
\hline
\end{tabular}

Fuente: Elaboración propia a partir de los resultados del estudio $\mathrm{n}^{\circ} 2.848 \mathrm{del} \mathrm{CIS}$

* Valor de Chi cuadrado de Pearson 9,757; significación asintótica bilateral 0,008

** Valor de Chi cuadrado de Pearson 18,183; significación asintótica bilateral 0,000 
La pregunta sobre el grado de acuerdo con la afirmación "después de cumplida la condena, debería ser devuelta la custodia al padre" añade un elemento de permanencia en el tiempo que no incluía el supuesto anterior, ligado a la sentencia. El resultado para el conjunto de la muestra vuelve a situar el porcentaje de mujeres que parecen asociar la violencia contra la pareja con el rol parental del agresor en torno al $65 \%$ (que es el porcentaje de encuestadas que está poco o nada de acuerdo con la devolución de la custodia al padre una vez cumplida la condena). Sólo un $12 \%$ está muy o bastante de acuerdo con la afirmación anterior, si bien destaca el aumento de las mujeres que no saben o simplemente optan por no dar respuesta a esta pregunta (en torno al $20 \%$ ).

Los resultados que ofrece la tabla 5 muestran cierta coherencia con los que podíamos observar en la tabla 4. Así, las mujeres víctimas de violencia tipo A son en mayor medida, y de forma significativa, partidarias de devolver la custodia al padre una vez éste ha cumplido la condena. Sin embargo, las mujeres que se han sentido maltratadas por una pareja o expareja alguna vez en su vida (violencia tipo B) son las que expresan un mayor rechazo ante esta devolución (en torno a un $74 \%$ está poco o nada de acuerdo). En todo caso, existe una relación significativa negativa entre ambas formas de violencia y la categoría $\mathrm{NS} / \mathrm{NC}$, de manera que las mujeres víctimas tanto de violencia tipo $A$ como tipo $B$ expresan o parecen tener más clara su posición. En contraposición, la existencia de una condena ya cumplida plantea mayores dudas entre las mujeres que, en principio, no padecen violencia.

TABLA 5. Tabla de contingencia entre violencia (tipo A y tipo B) y opinión sobre la devolución de custodia después de cumplida la condena (\%)

\begin{tabular}{|l|l|l|l|l|l|}
\hline \multicolumn{2}{|c|}{} & \multicolumn{2}{l|}{ Violencia tipo $A^{*}$} & \multicolumn{2}{l|}{ Violencia tipo $B^{* *}$} \\
\cline { 3 - 6 } & No & Sí & No & Sí \\
\hline \multirow{3}{*}{$\begin{array}{l}\text { Grado de acuerdo con la afirmación: } \\
\text { "Después de cumplida la condena, } \\
\text { debería ser devuelta la custodia al }\end{array}$} & \multirow{2}{*}{$\begin{array}{l}\text { Mucho/Bastante } \\
\text { padre" }\end{array}$} & 11,6 & 14,5 & 12,1 & 10,1 \\
& & $(-2,4)$ & $(2,4)$ & $(1,7)$ & $(-1,7)$ \\
\cline { 2 - 6 } & \multirow{2}{*}{ NS/NC/Nada } & 64,7 & 66,7 & 63,9 & 73,5 \\
& $(-1,1)$ & $(1,1)$ & $(-5,6)$ & $(5,6)$ \\
\hline Total & 23,7 & 18,8 & 24,0 & 16,4 \\
& $(3,1)$ & $(-3,1)$ & $(5,0)$ & $(-5,0)$ \\
\hline
\end{tabular}

Fuente: Elaboración propia a partir de los resultados del estudio $\mathrm{n}^{\circ} 2.848 \mathrm{del} \mathrm{CIS}$

* Valor de Chi cuadrado de Pearson 12,884; significación asintótica bilateral 0,002

** Valor de Chi cuadrado de Pearson 32,377; significación asintótica bilateral 0,000 


\section{Conclusiones}

A lo largo de este artículo se ha llevado a cabo un análisis secundario de los datos ofrecidos por el estudio $n^{\circ}$ 2.848 del CIS, que constituye la cuarta macroencuesta realizada en España sobre violencia contra las mujeres. De esta forma, y teniendo en cuenta las variables disponibles, hemos intentado analizar: 1) la presencia de menores en los casos de VCl; 2) la posible relación entre esta presencia y la formulación de denuncias por parte de la mujer; y 3) la percepción que la encuestada tiene del rol parental del agresor.

Por lo que respecta a la presencia de hijos menores en los casos de violencia tipo A (mujeres calificadas técnicamente como maltratadas) y tipo B (mujeres autoclasificadas como maltratadas), podemos concluir que ésta es relativamente elevada. Así, en un $54 \%$ de los casos de violencia tipo B y en un $43 \%$ de los de tipo A había hijos menores expuestos a la misma. Entre las mujeres que se han sentido maltratadas alguna vez en su vida (violencia tipo B) y tenían hijos menores en el momento del maltrato, un $53 \%$ afirma que sus hijos también han padecido directamente situaciones de violencia. Aun siendo elevado el porcentaje, debemos interpretarlo a la luz de los resultados de autores previos que destacan una tendencia entre las madres a subestimar el grado de

${ }^{48} \varnothing$ VERLINE, 2010, p. 89.

${ }^{49}$ Åsa CATER y Anna M. FORSELL, 2014; Maria ERIKSSON y Elisabet NÄSMAN, 2008; Carolina $\varnothing$ VERLIEN y Margareta HYDÉN 2009.

${ }^{50}$ ESPINAR, 2006. implicación de sus hijos en la violencia ${ }^{48}$. De esta forma, es necesario el desarrollo de nuevas investigaciones en dos ámbitos: por un lado, en la búsqueda de fuentes alternativas de información más allá de las progenitoras, como puedan ser los propios niños y niñas ${ }^{49}$; por otro lado, en el análisis de la interpretación que las mujeres hacen de la violencia de género y del grado en que pueda afectar a los menores expuestos a ella.

La primera de las hipótesis que nos planteamos en esta investigación preveía que la presencia de hijos en una relación donde se produce violencia de género condiciona a la mujer de manera positiva a la hora de efectuar la denuncia. Sin embargo, los resultados obtenidos parecen respaldar investigaciones previas que destacan el papel contradictorio que juega la presencia de $\operatorname{hijos}^{50} \mathrm{y}$, por tanto, sólo permiten una confirmación parcial de la hipótesis. Así, aunque la mayor parte de las mujeres que se han sentido maltratadas alguna vez en su vida (violencia tipo B) no interpusieron denuncia alguna; la presencia de hijos sí parece correlacionar con una mayor probabilidad de denunciar. Ahora bien, los hijos son también una de las principales razones que las mujeres plantean al ser cuestionadas por la no formulación de denuncias. En este sentido, sería necesario analizar otros aspectos como es no sólo la posibilidad de denunciar, sino también la de poner fin a una relación de 
${ }^{51}$ ERIKSSON, 2002; GUILLE, 2004; PEREL Y PELED, 2008; ROTHMAN MANDEL Y SILVERMAN 2007 SALISBURY, HENNING y HOLDFORD, 2009.

${ }^{52}$ Åsa CATER, 2004.

pareja marcada por la violencia; o la interpretación que la mujer hace respecto a la convivencia de los hijos con el agresor. Para este último análisis sería especialmente útil la aplicación de técnicas cualitativas de investigación.

Respecto a la segunda hipótesis, preveíamos una tendencia a disociar la violencia hacia la pareja del rol paterno del agresor, de manera que la presencia de actitudes o comportamientos violentos hacia la madre no supondría, necesariamente, una desvalorización de las habilidades parentales del agresor ${ }^{51}$. Sin embargo, los resultados obtenidos no parecen respaldar esta hipótesis. Así, es relativamente elevado el porcentaje de mujeres (en el conjunto de la muestra) que se pronuncia en desacuerdo con las afirmaciones: "Que un hombre maltrate a su pareja no tiene por qué implicar que sea un mal padre" y "Después de cumplida la condena, debería ser devuelta la custodia al padre". Especialmente elevado es el porcentaje de mujeres que afirman estar bastante o muy de acuerdo con la afirmación "Si hay sentencia condenatoria firme se debe quitar la custodia de los menores al maltratador".

Llama la atención que sean las mujeres víctimas tanto de violencia tipo A como de violencia tipo B (en comparación con el resto de mujeres) las que en mayor medida y de forma significativa se muestran de acuerdo con el hecho de que maltratar a la pareja no convierte al agresor en mal padre (parece, de esta forma, que son las más partidarias de disociar el rol de pareja-agresor del de progenitor). Sin embargo, cuando en la posibilidad del maltrato se incluye la existencia de condenas por violencia, los resultados varían. Las mujeres víctimas de violencia parecen tener opiniones más claras (con porcentajes menores en las categorías No sabe/No contesta) que el resto de la muestra. Por otra parte, si bien las víctimas de violencia tipo A siguen mostrando una mayor tendencia a disociar los roles, las víctimas de violencia tipo B se muestran más críticas con el papel de los agresores como padres, con un mayor rechazo a que puedan mantener la custodia de los menores. Estos datos nos llevan a plantear la necesaria profundización en el estudio de una serie de aspectos como es la propia percepción por parte de las mujeres de las diferentes formas de violencia, de la posible exposición de sus hijos y de la relación de éstos con sus padres.

En definitiva, se trata de un campo de estudio que requiere todavía de un gran trabajo de investigación, especialmente de naturaleza cualitativa, y centrado en las propias vivencias e interpretaciones de los menores involucrados, tomando a éstos directamente como informadores ${ }^{52}$. Igualmente, es necesario incidir en el análisis de la interpretación que las mujeres hacen de la violencia, 
de los riesgos para sus hijos y del rol parental del agresor ${ }^{53}$; así como en el estudio de la percepción que tienen los propios agresores en torno a su rol parental y a la relación que mantienen con sus hijos ${ }^{54}$. Todo ello ámbitos de necesaria profundización para la comprensión y actuación con los niños y niñas expuestos a VCl.

\section{Bibliografía}

ANNERBACK, Eva M.; WINGREN, Gun; SVEDIN, Carl G.; GUSTAFSSON, Per A. "Prevalence and characteristics of child physical abuse in Sweden: findings from a population-based youth survey”. Acta Paediatrica, v. 99, n. 8, p. 1229-1236. 2010.

ARAUJO, Kathya; GUZMÁN, Virginia; MAURO, Amalia. "Surgimiento de la violencia doméstica como problema público y objeto de políticas". Revista de la CEPAL, n. 70, p. 133145. 2000.

ATENCIANO, Beatriz. "Menores expuestos a violencia contra la pareja: notas para una práctica clínica basada en la evidencia". Clínica y Salud, v. 20, n. 3, p. 261-272. 2009.

AYLLON, Elena (coord.). En la violencia de género no hay una sola víctima: Atención a los hijos e hijas de mujeres víctimas de violencia de género. Save the Children, 2011. Disponible en: <http://www.savethechildren.es>. Acceso en: $19^{\circ}$ feb. 2014.

BALLIF-SPANVILL, Bonnie; CLAYTON, Claudia J.; HENDRIX, Suzanne B.; HUNSAKER, Melanie. "Individual Differences in the Use of Violent and Peaceful Behavior in Peer Conflicts among Children Who Have and Have Not Witnessed Interparental Violence". Journal of Emotional Abuse, v. 4, n. 2 , p. 101-123. 2004.

CATER, Åsa. Negotiating Normality and Deviation. Father's Violence against Mother from Children's Perspectives. Örebro (Suecia): Örebro University, 2004.

CATER, Åsa; FORSELL, Anna M. "Descriptions of fathers' care by children exposed to intimate partner violence (IPV): relative neglect and children's need". Child and Family Social Work, v. 19, n.2, p. 185-193. 2014.

CHANG, Judy C.; DADO, Diane; HAWKER, Lynn; CLUSS, Patricia; BURANOSKY, Raquel; SLAGEL, Leslie; MCNEIL, Melisa; SCHOLLE, Sarah $\mathrm{H}$. "Understanding turning points in intimate partner violence: Factors and circumstances leading women victims toward change". Journal of Women's Health, v. 19, n. 2, p. 251-259. 2010.

CUNNINGHAM, Alison; BAKER, Linda. What about me! Seeking to understand a child's view of violence in the family. London (Canadá): Centre for Children \& Families in the Justice System, 2004. 
EDIN, Kerstin; DAHLGREN, Lars; LALOS, Ann; HÖGBERG, Ulf. "'Keeping up a front': Narratives about intimate partner violence, pregnancy, and antenatal care". Violence Against Women, v. 16, n.2, p.189-206. 2010.

ELMAN, R. Amy. "Gender Violence". In: WAYLEN, Georgina; CELIS, Karen; KANTOLA, Johanna; WELDON, S. Laurel (eds.). The Oxford Handbook of Gender and Politics. Oxford: Oxford University Press, 2013. p. 236-258.

ENANDER, Viveka; HOLMBERG, Carin. "Why does she leave? The leaving process(es) of battered women". Health Care for Women International, v. 29, n. 3, p. 200-226. 2008.

ERIKSSON, Maria. "Men's Violence, Men's Parenting and Gender Politics in Sweden". Nora. Nordic Journal of Women's Studies, v.10, n. 1, p. 6-15. 2002.

ERIKSSON, Maria."Children Who 'Witness' Violence as Crime Victims and Changing Family Law in Sweden". Journal of Child Custody, v. 7, n. 2, p. 93-116. 2010.

ERIKSSON, Maria. "Contact, shared parenting, and violence: children as witnesses of domestic violence in Sweden". International Journal of Law, Policy and the Family, v. 25, n. 2, p. 165-183. 2011.

ERIKSSON, Maria; HESTER, Marianne. "Violent men as goodenough fathers? A look at England and Sweden". Violence Against Women, v. 7, n. 7, p. 779-799. 2001.

ERIKSSON, Maria; NÄSMAN, Elisabet. "Participation in Family Law Proceedings for Children whose Father is Violent to their Mother". Childhood, v. 15, n. 2, p. 259-275. 2008.

ESPINAR, Eva. Violencia de género y procesos de empobrecimiento. Córdoba (España): Universidad de Córdoba, 2006.

ESPINAR, Eva; MATEO, Miguel Ángel. "Violencia de género: reflexiones conceptuales, derivaciones prácticas". Papers, n.86, p. 189-201. 2007.

FRA-European Union Agency for Fundamental Rights. Violence against women: an EU-wide survey. Main results. Luxemburgo: Oficina de Publicaciones de la Unión Europea. 2014. Disponible en: <http://fra.europa.eu>. Acceso en: $28^{\circ}$ mar. 2014.

GODDARD, Chris; BEDI, Gillinder. "Intimate Partner Violence and Child Abuse: A Child-Centred Perspective". Child Abuse Review, v. 19, n. 1, p. 5-20. 2010.

GRAHAM-BERMANN, Sandra A.; CASTOR, Lana E.; MILLER, Laura E.; HOWELL, Kathryn H. "The impact of intimate partner violence and additional traumatic events on trauma symptoms and PTSD in preschool-aged children". Journal of Traumatic Stress, v. 25, n. 4, p. 393-400. 2012.

GRIP, Karin. The Damage Done. Children Exposed to Intimate Partner Violence and their Mothers: Towards empirically based interventions in order to reduce negative health effects in children. Göteborg (Suecia): University of Gothenburg. 2012. 
GUILLE, Lara. "Men who batter and their children: An integrated review". Aggression and Violent Behaviour, v. 9, n. 2, p. 129-163. 2004.

HOLDEN, George W.; GEFFNER, Robert A.; JOURILES, Ernest N. Children Exposed to Marital Violence. Theory, Research and Applied Issues. Washington: American Psychological Association. 1998.

HOLT, Stephanie; BUCKLEY, Helen; WHELAN, Sadhbh. "The impact of exposure to domestic violence on children and young people: A review of the literature". Child Abuse and Neglect, v. 32, n. 8, p. 797-810. 2008.

JACKMAN, Mary R. "Gender, violence and harassment". In: SALTZMAN, Janet (ed.). Handbook of the Sociology of Gender. Nueva York: Springer, 2006. p. 275-317.

KUHLMAN, Kate Ryan; HOWELL, Kathryn H.; GRAHAM-BERMANN, Sandra A. "Physical health in preschool children exposed to intimate partner violence". Journal of Family Violence, v.27, n. 6, p. 499-510. 2012.

LEVENDOSKY, Alytia A.; BOGAT, G. Anne; MARTÍNEZ-TORTEYA, Cecilia. "PTSD symptoms in young children exposed to intimate partner violence". Violence Against women, v. 19, n. 2, p. 187-201. 2013.

LICHTER, Erika. L.; MCCLOSKEY, Laura. A. "The Effects of Childhood Exposure to Marital Violence on Adolescent Gender-role Beliefs and Dating Violence". Psychology of Women Quarterly, v. 28, n. 4, p. 344-57. 2004.

MARTÍN, Esperanza; MARTíN, Manuel. Las violencias cotidianas cuando las víctimas son las mujeres. Madrid: Instituto de la Mujer. 1999.

NSPCC. Child abuse and neglect in the UK today. Londres: NSPCC. 2012. Disponible en: <http://www.nspcc.org.uk>. Acceso en: $16^{\circ}$ may. 2014.

ØVERLIEN, Carolina. "Children Exposed to Domestic Violence: Conclusions from the Literature and Challenges Ahead". Journal of Social Work, v. 10, n. 1, p.80-97. 2010.

ØVERLIEN, Carolina; HYDÉN, Margareta. "Children's Actions when Experiencing Domestic Violence". Childhood. A Journal of Global Child Research, v. 16, n. 4, p. 479-96. 2009.

PELED, Einat. "Parenting by Men Who Abuse Women: Issues and Dilemmas". British Journal of Social Work, v. 30, n. 1, p. 25-36. 2000.

PEREL, Guy; PELED, Einat. "The fathering of violent men: Constriction and yearning". Violence Against Women, v. 14, n. 4, p. 457-482. 2008.

RENNER, Lynette M.; SLACK, Kristen Shook. "Intimate Partner Violence and Child Maltreatment: Understanding Intraand Intergenerational Connections". Child Abuse and Neglect, v. 30, n. 6, p. 599-617. 2006. 
RENZETTI, Claire M.; EDLESON, Jeffrey L.; BERGEN, Raquel K. Sourcebook on Violence against women (2nd edition). Thousand Oaks, CA: SAGE. 2011.

ROTHMAN, Emily. F; MANDEL, David G.; SILVERMAN, Jay G. "Abusers' perceptions of the effect of their intimate partner violence on children". Violence Against Women, v. 13, n. 11, p. 1179-1191. 2007.

RUIZ, Isabel; PLAZAOLA, Juncal; VIVES, Carmen; MONTERO, M. Isabel; ESCRIBÀ, Vicenta; JIMÉNEZ, Esther; MARTíN, David. "Variabilidad geográfica de la violencia contra las mujeres en España". Gaceta Sanitaria, v. 24, n. 2, p. 128-135. 2010.

SALAZAR, Mariano; HÖGBERG, Ulf; VALLADARES, Eliette; ÖHMAN, Ann. "The Supportive Process for Ending Intimate Partner Violence after Pregnancy: The Experience of Nicaraguan Women". Violence Against Women, v. 18, n. 11, p. 12571278. 2012.

SALISBURY, Emily J.; HENNING, Kris; HOLDFORD, Robert. "Fathering by Partner-Abusive Men. Attitudes on Children's Exposure to Interparental Conflict and Risk Factors for Child Abuse". Child Maltreatment, v. 14, n. 3, p. 232-242. 2009.

SIGMA DOS. III Macroencuesta sobre la violencia contra las mujeres. Informe de resultados. Madrid: Instituto de la Mujer.2006.

SMITH, Amy M.; O'LEARY, Susan. G. "Parent and partner violence in families with young children: Rates, patterns, and connections". Journal of Consulting and Clinical Psychology, v. 73, n. 3, p.435-444.2005.

WHO -World Health Organization. Global and regional estimates of violence against women: prevalence and health effects of intimate partner violence and nonpartner sexual violence, Ginebra: WHO. 2013. Disponible en: <http://www.who.int>. Acceso en: $20^{\circ}$ jun. 2013.

ZINZOW, Heidi. M.; RUGGIERO, Kenneth J; RESNICK, Heidi; HANSON, Rochelle; SMITH, Daniel; SAUNDERS, Benjamin. "Prevalence and mental health correlates of witnessed parental and community violence in a national sample of adolescents". Journal of Child Psychology and Psychiatry, v. 50, n. 4, p. 441-450. 2009.

[Recebido em 20 de maio de 2014 reapresentado em 13 de fevereiro de $/ 2015$ e aceito para publicação em 20 de fevereiro de 2015] 


\section{Childhood and Gender Violence: Quantification and Perceptions}

Abstract: A growing number of authors has been suggesting the necessary incorporation of children in the analysis of gender violence and, specifically, in the analysis of intimate partner violence against women (IPV). Such incorporation would be relevant not only for reducing children's invisibility and vulnerability, but also for achieving a better understanding of the characteristics and dynamics of IPV. Based on these considerations, in this article we present the results of a secondary analysis of the data obtained by the last Spanish macrosurvey on violence against women. The available information allows us to analyze: 1) the presence of children exposed to $I P V, 2)$ the relationship between this presence and women's decision to report the violence, and 3) women's perception about the parental role of the aggressor.

Key Words: Gender violence; Intimate partner violence; Childhood; Quantitative analysis; Parental role. 